\title{
LIDAR BATHYMETRIC EVALUATION BASED ON SCATTERING CLASSIFICATION ALGORITHM
}

\author{
Jian Gao ${ }^{1}$, Guoqing Zhou ${ }^{1}$, Haoyu Wang ${ }^{1}$, Xiang Zhou ${ }^{1,2, *}$, Yexuan Mu ${ }^{1}$, Shuhua Long ${ }^{1}$,Fan Yang ${ }^{1}$ \\ ${ }^{1}$ Guangxi Key Laboratory of Spatial Information and Geomatics, Guilin University of Technology, No. 12 Jian'gan Road, Guilin, \\ Guangxi 541004, China - gzhou@glut.edu.cn \\ ${ }^{2}$ Department of Mechanical and Control Engineering, Guilin University of Technology, No. 12 Jian'gan Road, Guilin, Guangxi
} 541004, China

KEYWORDS:Airborne lidar,Scattering classification algorithm,Bathymetry capability,Transmissivity,diffuse attenuation coefficient

\begin{abstract}
:
The evaluation of the bathymetric capability of traditional airborne lidar system is mostly based on the formula of bathymetric capability by evaluating the diffuse attenuation coefficient $\left(\mathrm{K}_{\mathrm{d}}\right)$. This method is derived form the assumption that the reflectance of sediment is fixed.In this study ,however,the reflectance of sediment is not fixed. Therefore, this study improves the ability of bathymetric formula, and proposes a particle scattering classification algorithm to obtain the transmissivity value. The algorithm filters the scattering modes of particles by scattering discrimination factor (q), and obtains the transmissivity values by using the scattering intensity formulas. Experiments show that, when the transmissivity is in the range of $0-1$ and the average values of $\mathrm{K}_{\mathrm{d}}(532 \mathrm{~nm})$ are $0.1150 \mathrm{~m}^{-1}, 0.0894 \mathrm{~m}^{-1}$ and $0.0903 \mathrm{~m}^{-1}$ in January, June and October respectively, accordingly, the bathymetric capabilities are $0-44 \mathrm{~m}, 0-61.5 \mathrm{~m}$ and $0-52.5 \mathrm{~m}$, respectively. Compared with the original bathymetric method, these results show that the maximum bathymetric value has measured by the improved bathymetric capability formula and scattering classification algorithm has decreased under the influence of the change of sediment reflectance, and the result is more consistent with the actual situation and more accurate.
\end{abstract}

\section{INTRODUCTION}

In recent years, airborne lidar has gradually developed into a major active underwater detection method(Qin et al., 2016). It can detect underwater by transmitting blue-green band laser that can penetrate water body(Zhou et al., 2009). It has the characteristics of high precision, high resolution, flexibility, high speed and efficiency, especially plays an important role in offshore bathymetry, coastal zone mapping and other fields. Therefore, the bathymetric capability of airborne lidar is a very important system parameter(Zhou et al., 2004, 2015).

Our research team won a key project of innovation-driven development in Guangxi in 2018. The purpose of this project is to develop an airborne laser high precision three-dimensional seabed measurement instrument (LiDAR). The principle and structure of the instrument are shown in Fig. 1. My task in this project is: underwater transmission model research and bathymetric evaluation of airborne lidar.

At present, people evaluate the diffuse attenuation coefficient $\left(\mathrm{K}_{\mathrm{d}}\right)$ of water body, and then evaluate the bathymetric ability of airborne lidar. The clearer the sea water is, the smaller the diffuse attenuation coefficient is, the easier the laser penetrates the sea water, and the larger the bathymetric value is (Qin et al., 2018). On the one hand, (Austin et al., 1986) or (Jamet et al., 2012,) have used the value of $\mathrm{K}_{\mathrm{d}}(532 \mathrm{~nm})$ obtained by remote sensing data inversion method to evaluate the bathymetric capability of airborne lidar. On the other hand, (Wang et al., 2003) or (Hu et al., 2018) have also evaluated the maximum bathymetric capability parameters of the system through the relationship between the simulated $K_{d}$ and the effective attenuation coefficient of the system. However, the evaluation of bathymetric capability based on the formula assumes that the effective receiving power of the system in the formula is constant. In fact, the reflectivity of sediment is not

Corresponding author:Xiang Zhou; E-mail: zqx0711@glut.edu.cn. fixed, so it has some limitations.

Firstly, this paper improves the original airborne lidar bathymetric capability formula, and obtains the bathymetric capability formula under the influence of two variables that the transmissivity $(\mathrm{T})$ reflecting the change of sediment reflectance and the diffuse attenuation coefficient $\left(\mathrm{K}_{\mathrm{d}}\right)$ reflecting the difference of water turbidity. Secondly, a scattering classification algorithm is proposed to obtain the transmissivity value according to the requirement of the improved bathymetric capability formula. The method filters the scattering modes of particles by scattering discrimination factor (q), and then calculates the high-precision transmissivity value according to Rayleigh scattering and Michaelis scattering intensity formulas respectively. Finally, transmissivity value obtained by this algorithm and the relatively fixed $\mathrm{K}_{\mathrm{d}}$ value obtained by the more mature inversion method are used to evaluate the bathymetric capability of the airborne lidar according to the improved depth capability formula.

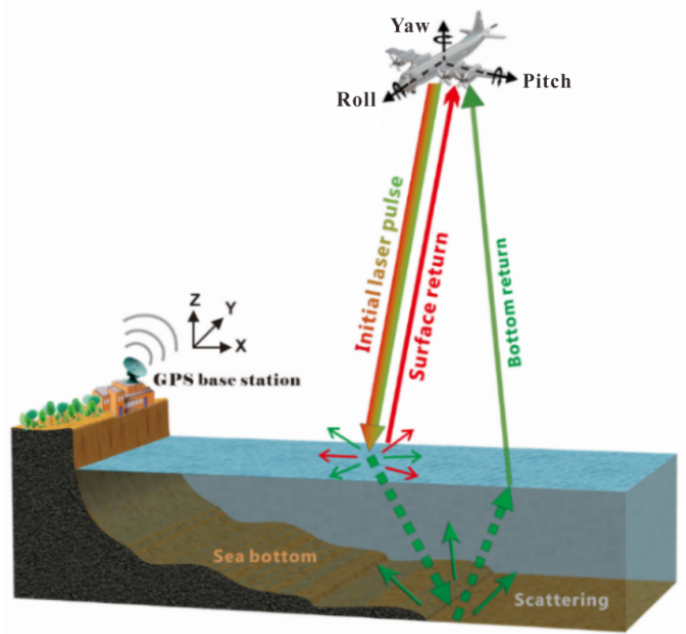

Fig 1: Dual LiDAR working diagram. 


\section{THEORY AND FORMULA IMPROVEMENT OF BATHYMETRIC CAPABILITY}

\subsection{Theoretical Basis of Bathymetric Capability}

The bathymetric capability formula of the original airborne lidar system is as follows(Steinvallo et al., 1981):

$$
\left\{\begin{array}{c}
\mathrm{L}_{\text {max }}=\frac{\ln \left(P_{A} / P_{B}\right)}{2 \mathrm{Q}} \\
P_{A}=\frac{P_{T} A_{r} \rho \eta}{\pi H^{2}}
\end{array}\right.
$$

In the formula, for $\mathrm{L}_{\max }$ is the maximum measurement depth, $\mathrm{Q}$ is the effective attenuation coefficient, $\mathrm{P}_{\mathrm{A}}$ is the effective receiving power, $\mathrm{P}_{\mathrm{B}}$ is the background noise power, $\mathrm{P}_{\mathrm{T}}$ is the peak power or the maximum emission power of the green laser, $\rho$ is the bottom reflectivity, $A_{r}$ is the effective receiving area of the laser receiver, $\eta$ is the efficiency of the receiving system, $\mathrm{H}$ is the altitude of the aircraft flying from the sea surface.

(Chen et al., 1996) established the relationship between the beam attenuation coefficient(c) and the effective attenuation coefficient $(\mathrm{Q})$ of the system by Monte Carlo method :

$$
\mathrm{Q}=\mathrm{c}\left(1-0.832 \mathrm{w}_{0}\right)
$$

In the formula, $\mathrm{c}$ denotes the attenuation coefficient of the beam and $\mathrm{W}_{0}$ denotes the single scattering albedo.

The relationship between the diffuse attenuation coefficient $\left(\mathrm{K}_{\mathrm{d}}\right)$ and the beam attenuation coefficient(c) obtained by (Fu et al., 2015) is as follows:

$$
\mathrm{K}_{\mathrm{d}} \cong \mathrm{c} \times\left[0.19\left(1-w_{0}\right)\right]^{\mathrm{w}_{0} / 2}
$$

Therefore, according to formulas (2) and (3), the relationship between the effective attenuation coefficient $\mathrm{Q}$ of the system and the diffuse attenuation coefficient $K_{d}$ is as follows:

$$
\mathrm{Q}=\frac{\mathrm{K}_{\mathrm{d}}\left(1-0.832 \mathrm{w}_{0}\right)}{\left[0.19\left(1-\mathrm{w}_{0}\right)\right]^{w_{0} / 2}}
$$

\subsection{Improvement of bathymetric capability formula}

Traditionally, when evaluating the bathymetric ability of airborne lidar system, it is considered that $\mathrm{P}_{\mathrm{A}}$ and $\mathrm{P}_{\mathrm{B}}$ are fixed in the formula(Formula 1) of airborne lidar bathymetric ability, so the bathymetric ability of airborne lidar depends on the effective attenuation coefficient $Q$ of its system.

According to formula (4) the relationship between the diffuse attenuation coefficient $\mathrm{K}_{\mathrm{d}}$ and the effective attenuation coefficient Q, Q is obtained through the evaluation of $K_{d}$, and then the bathymetric ability of airborne lidar is evaluated. In fact, the effective receiving power $\mathrm{P}_{\mathrm{A}}$ of the system varies with the change of the substrate reflectivity.The bottom reflectance varies with the different bottom sediments, and the bottom sediments can not be the same, which leads to some errors, so the bathymetric capacity formula is improved.

Laser energy is attenuated due to the absorption of dissolved impurities, water molecules and scattering of suspended impurities. The attenuation degree is closely related to the wavelength of the incident laser. The attenuation law obeys Beer-Lambert law (Zhang et al., 2007).

$$
\mathrm{I}_{\mathrm{x}}(\lambda)=I_{0}(\lambda) \exp [-\mathrm{c}(\lambda) x]
$$

In the formula, $\mathrm{I}_{0}(\lambda)$ is the initial irradiance or laser power of the laser before transmission, $\operatorname{Ix}(\lambda)$ is the irradiance of the laser after $x$-path transmission in the medium, and $c(\lambda)$ is the attenuation coefficient of the beam, which is the sum of absorption coefficient $a(\lambda)$ and scattering coefficient $b(\lambda)$. According to formula (5), the transmissivity of airborne lidar in seawater is defined as:

$$
\mathrm{T}=\mathrm{p} / \mathrm{p}_{0}=\exp [-\mathrm{c}(\lambda) \mathrm{x}]
$$

In the formula, $\mathrm{P}_{0}$ represents the power before the laser enters the water, $\mathrm{P}$ represents the power when the laser transmits a certain distance in the sea water, reflects through the seabed geology and receives by the receiver.

Transmissivity is defined as the ratio of actual receiving power to transmitting power. The theoretical value of the ratio of scattered light intensity to incident light intensity $\left(\mathrm{I} / \mathrm{I}_{0}\right)$ is the same as that of transmissivity. Further analysis shows that the ratio of effective receiving power $\left(\mathrm{P}_{\mathrm{A}}\right)$ to peak power $\left(\mathrm{P}_{\mathrm{T}}\right)$ of green laser in airborne lidar is the same as that of transmissivity. The transmissivity $\mathrm{T}$ and its relationship are obtained as follows:

$$
\mathrm{T}=\frac{\mathrm{P}}{\mathrm{P}_{0}}=\frac{I}{I_{0}}=\frac{\mathrm{P}_{\mathrm{A}}}{P_{T}}
$$

Therefore, the effective receiving power PA is expressed as:

$$
\mathrm{P}_{\mathrm{A}}=\frac{I}{I_{0}} \times \mathrm{P}_{\mathrm{T}}=\mathrm{T} \times \mathrm{P}_{\mathrm{T}}
$$

The peak power PT of green laser is fixed, so using this formula instead of effective receiving power $\mathrm{P}_{\mathrm{A}}$, we can control the error caused by the change of substrate reflectivity $\rho$ through transmissivity T, and further improve the accuracy. So by substituting formulas (4) and (8) into formulas (1), the improved formula of airborne lidar bathymetric capability is obtained as follows:

$$
\mathrm{L}_{\max }=\frac{\ln \left(\mathrm{T} \times \frac{P_{\mathrm{T}}}{P_{B}}\right)\left[0.19\left(1-\omega_{0}\right)\right]^{\omega_{0} / 2}}{2 \mathrm{~K}_{\mathrm{d}}\left(1-0.832 \omega_{0}\right)}
$$

The improved formula of bathymetric capability is influence under two variables that the transmissivity $\mathrm{T}$ to reflect the change of bottom reflectance and the diffuse attenuation coefficient $K_{d}$ to reflect the difference of turbidity of water body. Therefore, it is necessary to obtain the transmissivity value for evaluating the bathymetric ability of airborne lidar system. Next, the scattering classification algorithm for solving the transmissivity value will be described in this paper.

\section{SCATTERING CLASSIFICATION ALGORITHM}

\subsection{The Basis of Scattering Classification Algorithm}

The transmissivity of sea water can be considered as the ratio 
of the residual energy of laser after energy loss in water to the energy that was not lost before entering water. The energy loss of laser in seawater is mainly due to the scattering of particles in seawater. Light intensity is energy, so the value of transmissivity can be calculated according to the formula of scattered light intensity.

Scattering includes Rayleigh scattering and Michaelis scattering. There are many suspended particles such as impurities in coastal seawater, and the water quality is turbid. Therefore, Michaelis scattering is the main scattering in coastal waters, supplemented by Rayleigh scattering. In the traditional research on scattering, Rayleigh scattering is neglected mostly because the influence of Rayleigh scattering is less than several orders of magnitude. But in fact, the average concentration of suspended particulate matter near the coast is $80-250 \mathrm{mg} / \mathrm{L}$. According to the size of particles, the number of fine particles (radius 2-1000 nm) accounts for $90-98 \%$ of the total number of particles(Wang et al., 2015), and Rayleigh scattering accounts for a large proportion. Therefore, the influence of Rayleigh scattering is enlarged by several orders of magnitude, so the influence of Rayleigh scattering can not be ignored. Therefore, a scattering classification algorithm is proposed.

Based on the traditional theory, the scattering classification algorithm is designed to screen the scattering modes of particle of different particle sizes, and obtain the transmissivity value by using Rayleigh scattering and Michaelis scattering intensity formulas.

\subsection{Theory of Scattering Classification Algorithm}

Firstly, the scattering mode of particles in seawater is distinguished by calculating the value of scattering discrimination factor (q)(Howard $\mathrm{R}$ et al., 1982).

$$
\mathrm{q}=2 \pi R / \lambda
$$

$\mathrm{R}$ is the radius of the particle, $\lambda$ is the wavelength of the water-entering laser.

1) When the q value is less than 0.1, it is suitable for Rayleigh scattering. The characteristic of Rayleigh scattering is that the scattering intensity is inversely proportional to the fourth power of the wavelength, that is to say, the longer the wavelength is, the weaker the scattering is. When the scattering in all directions is weak, the transmissivity of the laser in the original propagation direction will be stronger. The scattering intensity of a single spherical particle is as follows(James H et al., 2017):

$$
\mathrm{I}=\frac{\mathrm{I}_{0}}{1^{2}} \frac{16 \pi^{4} \mathrm{R}^{6}}{\lambda^{4}}\left[\frac{n^{2}-1}{n^{2}+2}\right]^{2}\left(1-\sin ^{2} \theta \cos ^{2} \Phi\right)
$$

The transmissivity at this time is:

$$
\mathrm{T}=(1-\mathrm{I}) / \mathrm{I}_{0}=1 / \mathrm{I}_{0}-\frac{1}{1^{2}} \frac{16 \pi^{4} \mathrm{R}^{6}}{\lambda^{4}}\left[\frac{n^{2}-1}{n^{2}+2}\right]^{2}\left(1-\sin ^{2} \theta \cos ^{2} \Phi\right)
$$

In the formula, the optical refractive index of the scattering particle is $\mathrm{n}$, the radius is $\mathrm{R}$, the distance between the scattering particle and the observation point is $1(1$ is far larger than the radius of the particle), the incident light is linearly polarized and the wavelength is $\lambda$, the incident light intensity is $I 0$, and the scattering angle is $\theta$ (Zhong et al., 2010).

$$
\theta=\arccos \left\{\frac{1}{2 g}\left[\left(1+g^{2}\right)-\frac{\left(1-g^{2}\right)}{\left(1+g-2 g r_{1}\right)^{2}}\right]\right\}
$$

$\mathrm{r} 1$ is a random number with uniform distribution between $[0,1]$, $\mathrm{g}$ is an asymmetric factor, and generally 0.85 is chosen according to experience.Scattering azimuth is $\varphi$.

$$
\Phi=2 \pi \times \mathrm{r}_{2}
$$

Azimuth $\varphi$ is a uniform distribution on $(0,2 \pi), \mathrm{r} 2$ is a random number with uniform distribution between $[0,1]$.

When the q value is greater than 0.1, it is suitable for Michaelis scattering. When the diameter and wavelength of particles in seawater are almost equal, the scattering is called Michaelis scattering. The scattering of suspended particles in sea water is analyzed by Michaelis scattering theory. The scattering intensity of suspended particles is as follows(James $\mathrm{H}$ et al., 2017):

$$
\mathrm{I}=\frac{\lambda^{2}}{8 \pi^{2} l^{2}} I_{0}\left[i_{1} \sin ^{2} \varphi+i_{2} \cos ^{2} \theta\right]
$$

Among them, I1 is the vertical component of scattered light and I2 is the parallel component(Lai et al., 2010).

$$
\begin{gathered}
i_{1}=\frac{I_{0}}{l^{2}} \frac{16 \pi^{4} \mathrm{R}^{6}}{\lambda^{4}}\left(\frac{n^{2}-1}{n^{2}+2}\right) \\
i_{2}=\frac{I_{0}}{l^{2}} \frac{16 \pi^{4} \mathrm{R}^{6}}{\lambda^{4}}\left(\frac{n^{2}-1}{n^{2}+2}\right)^{2} \cos ^{2} \theta
\end{gathered}
$$

The transmissivity in this case:

$$
\mathrm{T}=(1-\mathrm{I}) / I_{0}=1 / \mathrm{I}_{0}-\frac{\lambda^{2}}{8 \pi^{2} l^{2}}\left[i_{1} \sin ^{2} \varphi+i_{2} \cos ^{2} \theta\right]
$$

The flow chart of scattering classification algorithm is shown in Fig 2.

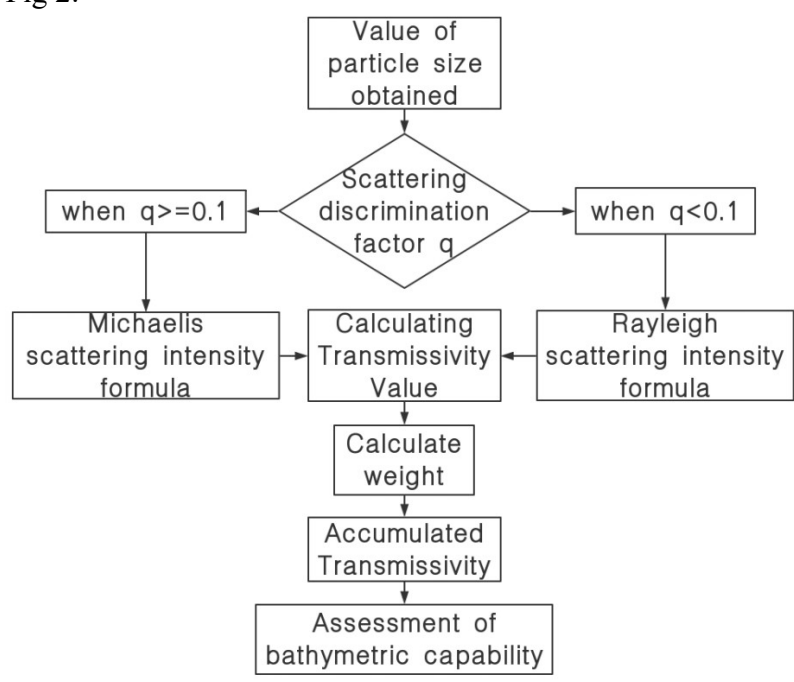

Fig. 2 Flow chart of scattering classification algorithm

Random numbers are generated to simulate the particle sizes of different particle and distributions in seawater. In the scattering classification algorithm, the scattering of light by each particle is calculated separately, so the weight $\left(\mathrm{W}_{\mathrm{i}}\right)$ of the transmissivity 
obtained is 1 . Therefore, the transmissivity value of a certain sea area can be obtained only by accumulating the values of the transmissivity obtained by each particle. That is:

$$
\mathrm{T}=\sum_{1}^{\mathrm{n}} T_{i} \times \mathrm{W}_{\mathrm{i}}
$$

In conclusion, when the particle size distribution in the sea area to be measured is known in advance, it can be used in the scattering classification algorithm to simulate the transmissivity value through the algorithm. Then the relatively fixed values of $\mathrm{K}_{\mathrm{d}}$ can be obtained by more mature inversion methods, which can be brought into the improved bathymetric capability formula to evaluate the bathymetric capability of airborne lidar system to sea area more accurately and quickly. At present, there are two methods to obtain particle size, one is the Kurt counter measurement method, the other is the empirical method of particle size. This article does not elaborate.

\section{EXPERIMENTS AND RESULTS}

\subsection{Simulation experiment}

\subsection{1 comparison experiments of Transmissivity}

The parameters of the airborne lidar system are CZMIL bathymetric system of Optech $\operatorname{Co}($ (Lai et al., 2010)). The environmental parameters are taken from the typical parameters of the airborne lidar bathymetric system and seawater quality. The main parameters of the simulation experiment are as follows:

Table 1:Main parameters of simulation experiment

\begin{tabular}{cc}
\hline parameter & Explain \\
\hline Lidar $/ \lambda$ & Green $532 \mathrm{~nm}$ \\
Laser Emission Power $/ \mathrm{I}_{0}$ & $0.5 \times 1015 \mathrm{w}$ \\
Refractive indexn & 1.34 \\
Distance Between Scattered & \\
Particles and Observation & $1 \times 1015 \mathrm{~nm}$ \\
Points/1 & 0.85 \\
Asymmetric factor/g & Uniform Distribution Random \\
$\mathrm{r}_{1,2}$ & Numbers between 0 to 1 \\
Particle radius $/ \mathrm{R}$ & Representation by generated \\
Sunlight background noise & random numbers \\
power $/ \mathrm{P}_{\mathrm{B}}$ & $>4.3 \times 10-8 \mathrm{w}$ \\
Aircraft flight altitude/m & $400 \mathrm{~m}$ \\
single scattering albedo/ $/ \mathrm{w}_{0}$ & 0.7 \\
diffuse attenuation & $0.0894 、 0903$ and $0.1150 \mathrm{~m}^{-1}$ \\
coefficient $/ \mathrm{K}_{\mathrm{d}}$ &
\end{tabular}

Based on the theory of scattering classification algorithm, the transmissivity is simulated by using MATLAB software. Since Rayleigh scattering is mostly neglected in the study of the influence of traditional laser propagation underwater, the first step is to verify whether Rayleigh scattering can be neglected. Firstly, the simulation and comparison experiments of transmissivity based on the scattering classification algorithm and considering only the influence of Michaelis scattering (i.e. ignoring Rayleigh scattering) are carried out. According to the traditional rule of comparative experiment, the effect of pure Rayleigh scattering is added to the experiment to verify the effectiveness of the algorithm.

Firstly, the same random number is generated each time in the Matlab software to control the influence caused by the change of random number.In the range of $1-1000 \mathrm{~nm}, 107$ random numbers are generated as the size of particle size in seawater. Secondly, the particle size distribution is that the number of particles producing Rayleigh scattering is more than two orders of magnitude higher than the number of particles producing Michaelis scattering.It is more practical to use this distribution as the distribution of particle size in seawater. Finally, these random numbers are brought into the simulation program to calculate the transmissivity value. Because of the contingency of one simulation, 10 simulation experiments were carried out, and their values were fitted and used as comparative experiments. The results are shown in Fig 3.

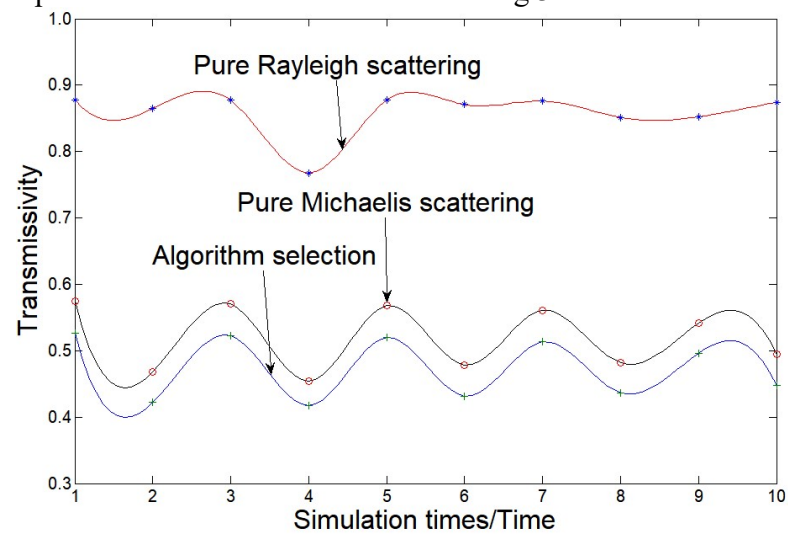

Fig 3 Transmissivity contrast diagram based on scattering classification algorithms

According to the simulation and comparison experiments of transmissivity, there are some differences between the filtered transmissivity of the scattering classification algorithm and the transmissivity under the influence of pure Michaelis scattering. This difference is due to the consideration of Rayleigh scattering, so Rayleigh scattering can not be ignored, and the algorithm is effective. Under the same simulation, the transmissivity under the influence of pure Rayleigh scattering is the highest, so its influence on bathymetric is the smallest, which accords with the theory, and thus further proves the effectiveness of the algorithm.

\subsubsection{Transmissivity simulation experiment}

In order to simulate the transmissivity of particles with different sizes and distributions, the random numbers of different proportions at different sizes are controlled as the distribution of particles producing Rayleigh scattering or Michaelis scattering. Because the scattering discrimination factor $\mathrm{q}=2 \pi \times \mathrm{R} / \lambda$, where $\lambda$ is $532 \mathrm{~nm}$, it is inversely calculated that Michaelis scattering will occur when $\mathrm{R}$ is greater than $8.4 \mathrm{~nm}$ and Rayleigh scattering will occur when $\mathrm{R}$ is less than $8.4 \mathrm{~nm}$. Therefore, the ratio of Rayleigh scattering or Michaelis scattering can be controlled by controlling the number of random numbers between $1 \sim 8.4 \mathrm{~nm}$ and $8.4 \sim 1000 \mathrm{~nm}$. 
The rand() program is used to generate $10^{7}$ random numbers with uniform distribution in Matlab software. As the size of particles in seawater, the range is 1-8.4 and $8.4-1000 \mathrm{~nm}$, respectively. When Michaelis scattering is $1 \%$ and $6.5 \%$ respectively, the transmissivity is simulated. To eliminate contingency, we performed 20 simulations. The scatter plot of tansmissivity values of $1 \%$ and $6.5 \%$ Michaelis scattering are shown in figs. 4 and 5.

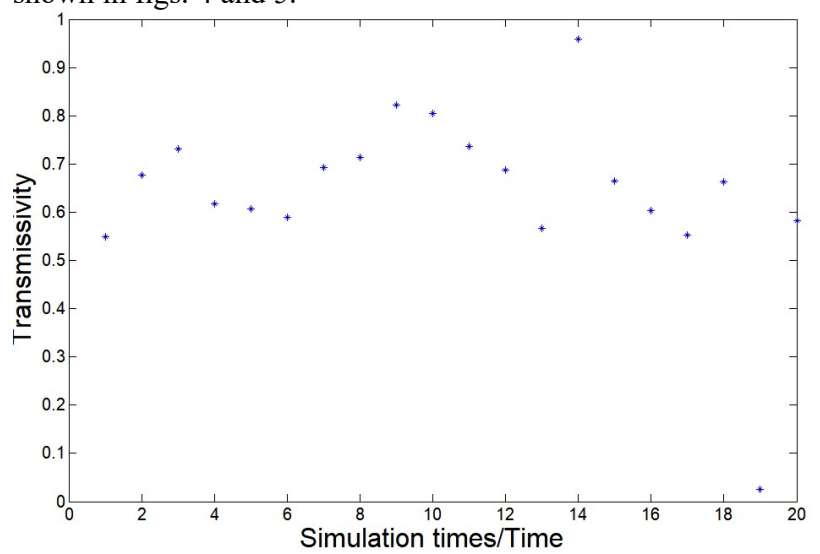

Fig 4 Transmissivity simulation of $1 \%$ Michaelis scattering

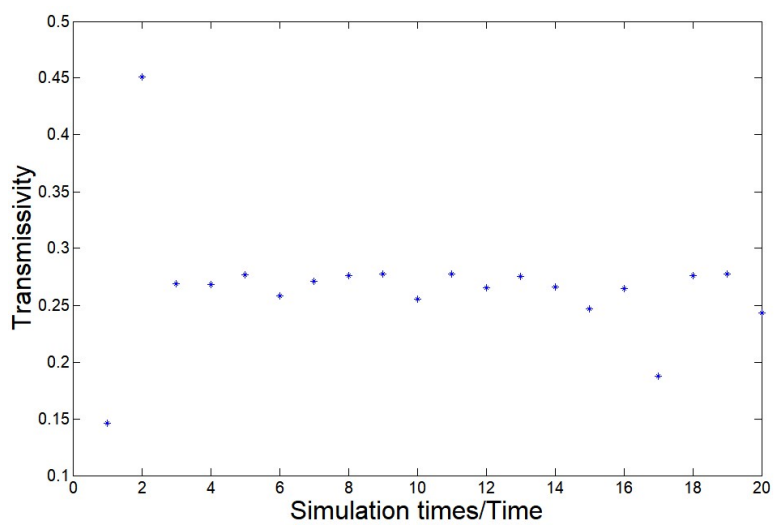

Fig 5 Transmissivity simulation of $6.5 \%$ Michaelis scattering

Furthermore, we use the proportion of Michaelis scattering as an independent variable to simulate the transmissivity and and use the cubic spline interpolation method to make the fitting image. The simulation figure of transmissivity under Michaelis scattering ratio is shown in Fig. 6.

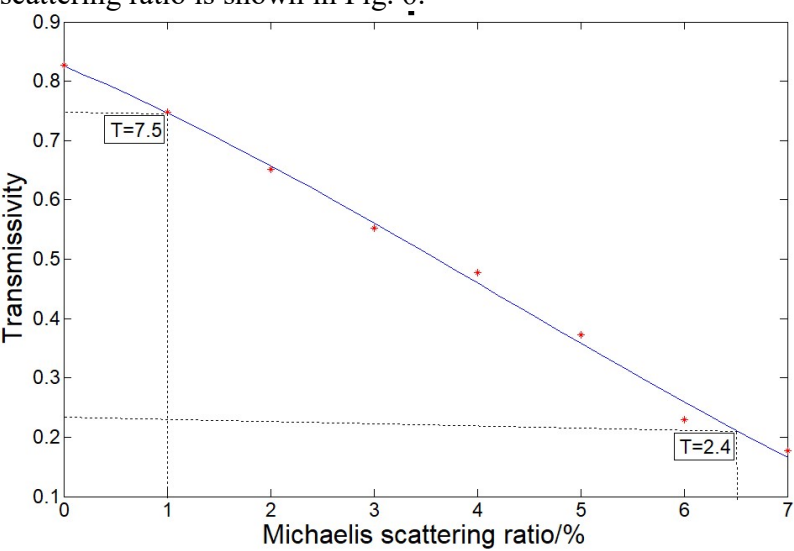

Fig 6 Transmissivity simulation under Mie scattering ratio

From the simulation results of the transmissivity of $1 \%$ and $6.5 \%$ Michaelis scattering, it can be seen that the transmissivity only varies between 0.5 and 0.8 when Michaelis scattering is $1 \%$, and between 0.2 and 0.3 when Michaelis scattering is $6.5 \%$. It is concluded that the values of multiple simulated transmissivity change almost in a certain range when the Mie scattering ratio is constant. Therefore, the experimental values have certain stability and accuracy.

Fig. 6 shows that with the increase of Michaelis scattering from $0 \%$ to $7 \%$, the transmissivity decreases gradually, almost linearly, and the transmissivity values of Michaelis scattering at $1 \%$ and $6.5 \%$ are in accordance with the results of Fig. 4 and Fig. 5. Therefore, we obtain the relative accurate range of the transmissivity value under different proportion of Michaelis scattering.

\subsection{The simulation experiment of Lidar bathymetric ability}

The bathymetric ability of airborne lidar under different transmissivity is more objective. Therefore, taking the northern South China Sea as an example, when the transmissivity is between 0 and 1,the bathymetric capability of airborne lidar is simulated in Matlab by using satellite-borne sensor remote sensing inversion $\mathrm{K}_{\mathrm{d}}$ value and optimized bathymetric capability formula.

The diffuse attenuation coefficient $K_{d}$ is the value of the northern South China Sea retrieved by spaceborne sensors by ( $\mathrm{Li}$ et al., 2018a and 2018b). The average values of $\mathrm{K}_{d}$ in January, June and October are $0.1150 \mathrm{~m}^{-1}, 0.0894 \mathrm{~m}^{-1}$ and $0.0903 \mathrm{~m}^{-1}$, respectively.

The simulation results in MATLAB software show that the bathymetric capabilities in January, June and October of the northern South China Sea are $0 \sim 44 \mathrm{~m}, 0 \sim 61.5 \mathrm{~m}$ and $0 \sim 52.5 \mathrm{~m}$, respectively. June is the best month for water quality, so compare the results of June. (Li et al., 2018a or 2018b) used the original method to evaluate the measurable water depth of the northern South China Sea in June, which is about $0-71.5 \mathrm{~m}$. However, under this method, the measurable water depth of the northern South China Sea in June is about $0-62.5 \mathrm{~m}$. It is concluded that the maximum bathymetric value evaluated by this method has been reduced, because the variation of sediment reflectance and the influence of Rayleigh scattering have been taken into account in this paper. Therefore, the accuracy of the results is higher. The Response Chart of the depth of measurement with the change of transmissivity is shown in Fig. 7.

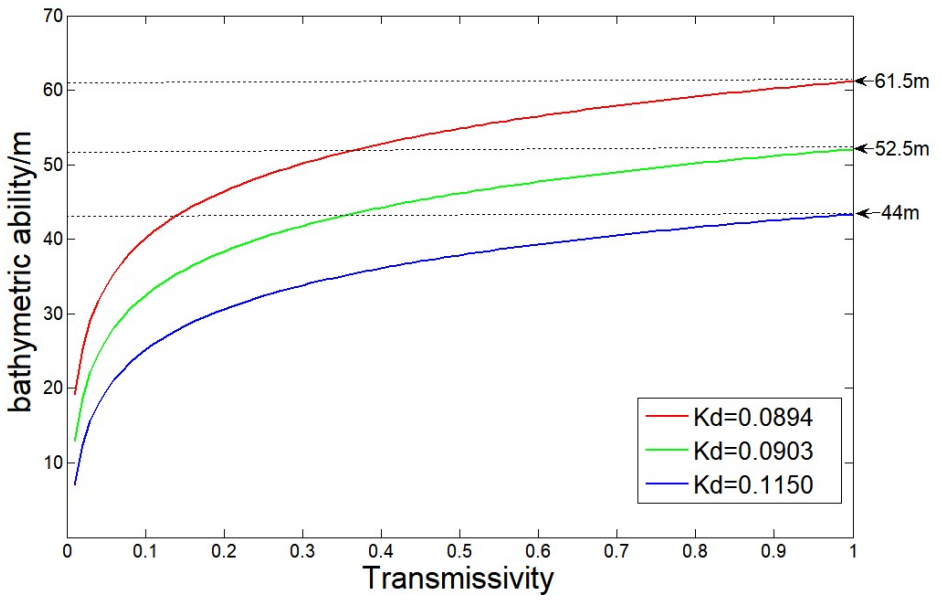

Fig 7 Response chart of measured depth with transmissivity 


\section{CONCLUSIONS}

In this paper, the transmissivity value is simulated by MATLAB software, and the Bathymetric maximum of airborne lidar is simulated according to the relatively fixed $\mathrm{K}_{\mathrm{d}}$ and the improved formula of bathymetric ability. The following conclusions are drawn:

The filtered transmissivity of the scattering classification algorithm is slightly lower than that of Michaelis scattering only. This difference is due to the neglect of Rayleigh scattering, so the algorithm is effective.

From the simulated transmissivity values of $1 \%$ and $6.5 \%$ Michaelis scattering, it can be seen that when the Michaelis scattering proportion is constant, the simulated results change almost in a small range. Therefore, the stability and accuracy of the algorithm are obtained.

With the increase of Michaelis scattering ratio from $0 \%$ to $7 \%$, the transmissivity decreases gradually and almost linearly. Therefore, in a certain range, the transmissivity value decreases gradually with the increase of Michaelis scattering proportion.

Under this evaluation method, the measured water depth is $0-61.5 \mathrm{~m}$. Comparisons show that the maximum bathymetric value of this method has decreased, because this paper considers the variation of the reflectance of sediment and the influence of Rayleigh scattering. Therefore, the bathymetric results are more accurate.

\section{ACKNOWLEDGEMENTS}

This paper is financially supported by the National Natural Scie nce of China under Grant numbers 41431179,41961065; Guang xi Innovative Development Grand Grant under the grant numbe r GuikeAA18118038, GuikeAA18242048; the National Key Re search and Development Program of China under Grant numbe r 2016YFB0502501 and the BaGuiScholars program of Guang xi (Guoqing Zhou).

\section{REFERENCES}

Haiming Qin and Cheng Wang, et al. 2016a. Advances in Airborne Lidar bathymetric Technology and Application, Journal of Remote Sensing Technology and Applications, China,31(4), pp. 617-624.

Zhou, and Ming Xie, 2009. GIS-based Three-dimensional Morphologic Analysis of Assateague Island National Seashore from LIDAR Series Datasets, Journal of Coastal Research, vol. 25 , no. 2, pp. 435-447.

G. Zhou, C. Song, and W. Schickler, 2004. Urban 3D GIS from LIDAR and digital aerial images, Computers and Geosciences, vol. 30 , no. 4 , pp. 345-353.

G. Zhou, Xiang Zhou, Jiazhi Yang, Yue Tao, 2015. Xueqin Nong, Oktay Baysal, Flash Lidar Sensor using Fiber Coupled APDs , IEEE Sensor Journal, vol. 15, no. 9, September, pp. $4758-4768$

Haiming Qin, Cheng Wang, Xiaohuan Xi, Sheng Nie, and Guoqing Zhou, 2018. Integration of airborne LiDAR and hyperspectral data for maize FPAR estimation based on a physical model, IEEE Trans. On Geoscience and Remote Sensing Letter, Vol. 15, No. 7, July pp. 1120-1124.

Jamet C, Loisel H, Dessailly D. 2012. Retrieval of the Spectral Diffuse Attenuation Coefficient $\mathrm{K}_{\mathrm{d}}(\mathrm{)}$ in Open and Coastal Ocean Waters Using a Neural Network version. Journal of Geophysical Research: Atmospheres, 117(C10),:C130134.

Austin R W, Petzold T J. 1986. Spectral Dependence of the Diffuse Attenuation Coefficient of Light in Ocean Waters. Optical Engineering, 25(3). 253471.

Shanjiang $\mathrm{Hu}$, et al. 2018a. Design and Development of Airborne Dual-Frequency Lidar System. Journal of Infrared and Laser Engineering, China, 47(09), pp. 89-94.

Quandong Wang,Weibiao Chen,Yutian Lu,et al. 2003a. Parametric Design and Maximum Deep Detection Capability Analysis of Airborne Ocean Laser bathymetric System. Journal of Optics, China, 23(10).

Steinvallo, Klevebrant H, Lexander J,et al. 1981. Laser Depth Sounding in the Baltic Sea[J].Applied Optics, 20(19):3284-3286.

Wenge Chen, Benxiong Huang, Zongkai Yang, et al.1996a. Effective Attenuation Coefficient of Airborne Ocean Lidar System. Chinese Journal Of Electronics, China, 24(6):47-50.

Chengqun $\mathrm{Fu}$, Xiuyuan $\mathrm{Lv}$, Yong Wang, et al. 2015a. Simulating Research on Marine Detection of Airborne Lidar. Journal of System Simulation, China, 27(5):1038-1043.

Xiaochun Zhong, Yuanhui Li, et al. 2010a. Attenuation characteristics of laser in seawater. JJournal of University of Electronic Science and Technology, China, 39(4): 574-577.

Yingjian Wang, Chengyu Fan, 2015. Laser Propagation and Application in Atmosphere and Sea Water, National Defense Industry Press, :99-100

Howard R. Gordon, 1982. Interpretation of airborne oceanic lidar: effects of multiple scattering. Journal of Applied Optics / Vol. 21, No. 16 / 15 August.

James H. Churnside, Richard D. March banks, 2017. Optical Back scattering Measured by Airborne Lidarand Underwater Glider.Remote Sens. 9, 379; doi:10.3390/rs9040379.

Xiaochun Zhong, Yuanhui Li, 2010. Attenuation characteristics of laser in seawater, Journal of University of Electronic Science and Technology, 39(4): 574-577.

Xudong Lai, 2010. Basic Principle and Application of Airborne Lidar, Electronic Industry Press, :50-62

Kai Ding, Qingquan Li, Jiasong Zhu, et al. 2018a. Spectral Analysis of Water Diffuse Attenuation Coefficient and Estimation of LiDAR bathymetric Capability in the Coastal Area of Hainan Island. Journal of Spectroscopy and Spectral Analysis, China, 38(05):260-265.

Kai Ding, Qingquan Li, Jiasong Zhu, et al. 2018b. Evaluation of parameters of airborne lidar bathymetric system in northern South China Sea using MODIS remote sensing data. Journal of 
The International Archives of the Photogrammetry, Remote Sensing and Spatial Information Sciences, Volume XLII-3/W10, 2020 International Conference on Geomatics in the Big Data Era (ICGBD), 15-17 November 2019, Guilin, Guangxi, China

Surveying and mapping, China,47(2): 180-187. 